\title{
Microwave workshop for Windows
}

\author{
Colin White \\ Division of Physics, University of Portsmouth
}

This paper describes Microwave Workshop for Windows, a suite of three programs which have been designed in the Division of Physics in the University of Portsmouth to perform the dual function of teaching aid and providing a circuit-design utility in the field of microwave engineering.

\section{Introduction}

A suite of three programs has been developed to support the teaching of microwave theory and design. A secondary function of the package is to support microwave engineers by providing a library of utilities to assist their design function. All three programs were written in Visual Basic and are aimed at supporting both tutor-directed and student-centred learning methodologies. The development team consisted of three final-year degree students.

Program 1 is Smith Chart. The Smith Chart is one of the most important tools for the microwave engineer, yet students find the concepts of its application difficult to grasp using traditional teaching methods. Students can choose between running an animated tutorial or using the chart, in stand-alone mode, to solve problems.

Program 2 is Microwave Laboratory. This is a virtual microwave laboratory and includes all the waveguide components one would reasonably expect to see in such a laboratory. The components can be connected in any combination, and a generator and detector can then be connected to the completed circuit's remaining ports. Important parameters can be varied by the student, and results in terms of power or Voltage Standing Wave Ratio (VSWR) can be observed.

Program 3 is Microwave Device Technology, and is a hypertext interpretation of lecture course notes describing a wide range of devices at three levels of complexity. Level 1 


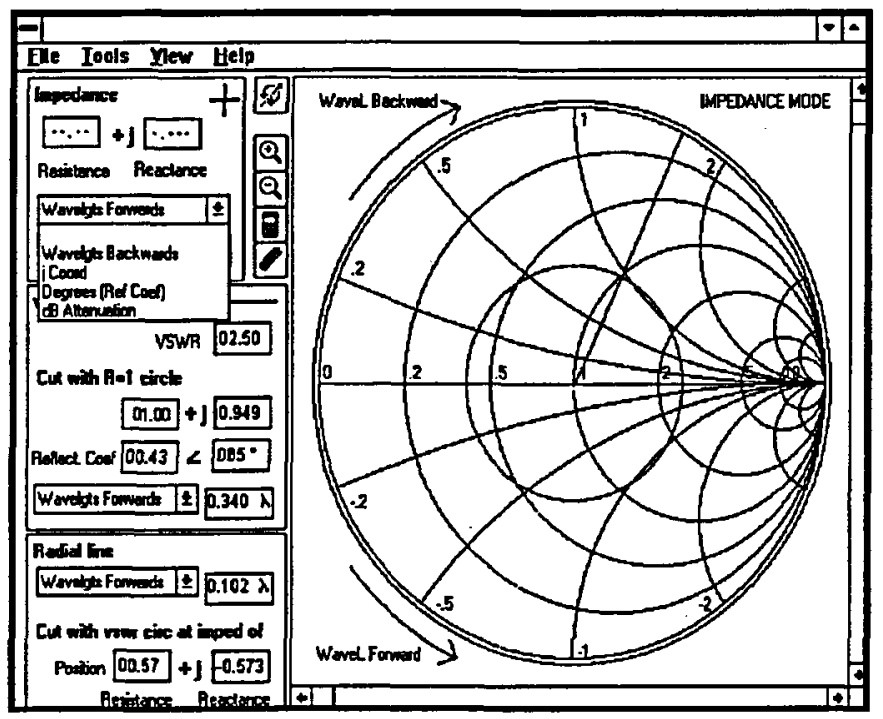

Figure 1: Smith Chart

reflects the requirements of a Higher Diploma unit, Level 2 corresponds to final-year degree level, and the highest level supports postgraduate teaching within the institution.

All three programs include extensive help files which describe not only program operation but also context-sensitive microwave theory and device descriptions.

\section{Smith Chart}

The currently accepted form of the Smith Chart was developed by Philip H. Smith in 1936, and is now extensively used by microwave and radio-frequency engineers to aid design work. The chart is a two-dimensional graph with real and imaginary axes (argand diagram) on which can be mapped complex impedance points such as those which may exist along a high-frequency cable or transmission line. The chart may be used to calculate the value of the impedance at the end of the line (the load impedance), or alternatively the length of a transmission line necessary to achieve an impedance match. The chart does not provide answers which could not be derived by someone of a good mathematical grounding armed with a calculator; rather, it performs the function of a special-to-type slide rule to speed up such calculations.

The individual steps required to perform the calculations using the chart are actually quite trivial. The difficulty students have in grasping the operations is, I believe, due in part to their inability to equate real and measurable quantities, such as voltage and power which exist along a transmission line, into coordinates and lines on the somewhat abstract concept of a chart. Indeed, some students successfully complete calculations on the chart by rote, with little knowledge of the underlying philosophy. The students' cognitive difficulty is further compounded by the fact that the two axes of the chart are circles and segments of circles respectively, and they are also highly non-linear. Finally, the confusion 
is exacerbated by virtue of one of the major attributes of the Smith Chart: each coordinate point represents firstly a complex impedance value, secondly the value of the reflection on the transmission line (the VSWR) and finally the distance of the point on the line from the line's termination. It is small wonder that students have trouble when faced with a real problem to solve, and given only a blank, black-and-white chart which is remote from any real hardware.

In such situations a coloured CAL animation becomes an extremely powerful learning tool. The Smith Chart tutorial takes the student in a step-wise manner from construction of the chart itself through to incremental problem solving. Students are at all times in control of the progression of the program, and hence their pace of study. Colour is used to highlight relevant areas of an otherwise invariable chart, and this is mirrored with highlighted points along a drawing of an actual transmission line. Movement around the chart corresponds to movement along the transmission line, and student-selectable animations are used to good effect to illustrate these aspects.

The Smith Chart utility is a fully operational chart on which all functions that would normally be carried out with a paper chart, pencil, ruler and compasses (and probably rubber!) can be performed by keyboard and mouse-click and displayed as accurately as the screen resolution will allow. A Magnify button is included for situations where screen resolution is a limiting factor. Results are as accurate as the computer's arithmetic precision has been set, rather than governed by the thickness of the pencil lead, and it is, consequently, orders of magnitude better than the traditional method. As the cursor is moved around the chart by the mouse, the corresponding impedance value is updated in real time. This dynamic nature, again, aids the students' understanding of the chart as they acquire a better feeling for the impedance values in different areas of the chart than they ever would by merely reading off the scales. Finally, although unnecessary in the normal course of events, a calculator button is included for emergencies.

Although this is not the only software implementation of the Smith Chart available, it is the only one designed primarily for teaching purposes. It is also the most intuitive and, perhaps most significantly, the only implementation which runs under Windows.

\section{Microwave Laboratory}

Microwave Laboratory is an accurate visual representation of a real teaching microwave laboratory. All the common components are represented. They are taken individually, as required, from the virtual component store, and drag-dropped onto the virtual test bench where they are automatically connected together if dropped adjacent to another component. In this manner, a complete circuit may be built up, and the input/output ports are connected to the microwave generator and receiver respectively. These, again, are accurate representations of commercially available equipment.

Devices capable of parameter variation are adjusted using a control pane launched by double-clicking the device. To aid the construction of an elegant arrangement of the circuit on the workbench, any device can be reoriented in $90^{\circ}$ steps by pressing the Rotate button. Further, any device can be deselected by dragging it to the Mac-style dustbin. 
Once a circuit has been constructed, the generator power and frequency can be selected and then switched on. First, the circuit is checked for validity by confirming that the workbench contains one launcher, one or two detectors, no open ports and no loops. Each device in the circuit defines a square array-type variable of order equal to the number of ports. This device array fully defines the device characteristics. A matrix multiplication routine calculates the power at the detector and displays it on the virtual Amp in digital and analogue format. If a standing wave detector has been selected, power is also displayed graphically in a pane below the workbench, as a function of length along the transmission line.

The usual Windows menus or short-cut keys allow any useful circuits to be saved and then opened at a future date or, indeed, to be printed.

Certain common devices are not available in the device store. There are, however, sound pedagogic reasons for this. By way of example, consider the wavemeter. This is a device used to measure very accurately, but with much time and effort, the output frequency of the generator. It is obvious that the frequency accuracy of the virtual generator is defined by the program's mathematical precision and is certainly orders of magnitude better than the real wavemeter. There is, therefore, no reason to specify a wavemeter and, perhaps more importantly, the measurement of accurate frequency can detract from the main aims of the experiment.

This laboratory simulation will model all the usual microwave experiments quickly, efficiently and accurately, but it important that students do not perceive it as the real world. The program is intended to support traditional experimental procedure but not to replace it. Typically, the student is given some time on the package, constructing the circuit and measuring relevant parameters before doing it for real. By this means he or she acquires an overview of what each device does, what happens when they are connected in a particular configuration, and the particular measurement technique to be learned by the experiment. An important part of the learning strategy is in the interpretation of measurement discrepancy between the virtual and the real laboratories.

\section{Microwave Device Technology}

The third program is simply a hypertext implementation of microwave course notes. The notes reside in three levels representing HND, final level degree and MSc teaching. The level can be switched at any point in the device descriptions to enable a lower-level student to read about a particular device at a higher level, if required (or even desired!). The program has been used in a traditional lecture environment using a laptop and overhead tablet as a cleaner alternative to chalk. Students have access to the notes on the network and they can print out any or all of the levels, as required, for study remote from a terminal.

\section{Future work}

Future work will involve both improvements to the existing programs and also additions of new programs to the workshop suite. 
The Smith Chart presently supports only calculations on one impedance point. Occasionally it is necessary to work with two points and, for instance, calculate the impedance difference. A future version will incorporate this facility.

The Microwave Laboratory can support only devices specified in the device store. It is a trivial addition to include a user-defined black box which may be connected on the workbench. In this case, by double-clicking on the black box, the user may write directly to the defining array the parameters of some device not included in the store:

However, a non-trivial addition to the microwave laboratory could be the inclusion of a swept-frequency generator and amplitude analyser. This would open up a range of more sophisticated experiments involving frequency response measurement techniques.

The Microwave Device program will soon have device diagrams reinforced by colour scanned images of the devices.

As for additional programs, a microstrip version of the laboratory simulator is planned which is thought to be of interest to commercial companies, and an antenna design CAL package and design utility. 